\title{
Coarsening rates for models of multicomponent phase separation
}

\author{
ROBERT V. KOHN ${ }^{\dagger}$ AND XIAODONG YAN ${ }^{\ddagger}$ \\ Courant Institute, 251 Mercer Street, New York, NY 10012, USA
}

[Received 2 July 2003 and in revised form 15 September 2003]

\begin{abstract}
We study the coarsening of solutions of two models of multicomponent phase separation. One is a constant mobility system; the other is a degenerate mobility system. These models are natural generalizations of the Cahn-Hilliard equation to the case of a vector-valued order parameter. It has been conjectured that the characteristic length scale $\ell(t)$ grows like $t^{1 / 3}$ as $t \rightarrow \infty$ for the first case and $\ell \sim t^{1 / 4}$ for the second case. We prove a weak one-sided version of this assertion. Our method follows a strategy introduced by Kohn and Otto for problems with a scalar-valued order parameter; it combines a dissipation relationship with an isoperimetric inequality and an ODE argument. We also address a related model for anisotropic epitaxial growth.
\end{abstract}

\section{Introduction}

We study the coarsening associated with two models of multicomponent phase separation, and a third model from the epitaxial growth literature. Each evolution has a scale-invariant sharp-interface limit, and it is natural to guess that coarsening proceeds at the "similarity rate." We prove a weak, one-sided version of this assertion: roughly speaking, the system can coarsen no faster than the similarity rate.

Our analysis uses the method introduced by Kohn and Otto in [22]. That paper discussed only binary systems, described by scalar-valued order parameters. This paper represents its natural generalization to multicomponent systems, described by vector-valued order parameters. The method of [22] relies upon (i) a dissipation relation, (ii) an isoperimetric inequality, and (iii) an $O D E$ lemma. The main novelty of the multicomponent setting is the isoperimetric inequality: its proof is quite different from the binary case.

The two models of multicomponent phase separation we shall consider are:

(A) Constant-mobility Cahn-Hilliard systems. Here the order parameter is $u=\left(u^{1}, \ldots, u^{m}\right) \in$ $\mathbb{R}^{m}$, and the PDE is

$$
u_{t}^{i}+\Delta^{2} u^{i}-\Delta\left(\nabla_{u^{i}} \Phi(u)\right)=0, \quad i=1, \ldots, m .
$$

It decreases the energy

$$
\int \frac{1}{2}|\nabla u|^{2}+\Phi(u)
$$

while conserving the mean value of $u$. The bulk term $\Phi(u)$ is nonnegative, vanishing at finitely many points which represent the system's distinct "phases." Equation (1) models the spinodal decomposition of an alloy with $m+1$ components (see e.g. [9, 10, 11, 15, 19, 27]). Typically $u^{i}$

\footnotetext{
†Email: kohn@cims.nyu.edu

‡Email: xiayan@math.msu.edu
} 
represents the scaled difference between the mass concentration of the $i$-th component and that of the $(m+1)$-th component. When $m=1$ and $\Phi(u)=\frac{1}{4}\left(u^{2}-1\right)^{2}$ the PDE reduces to the scalar Cahn-Hilliard equation $u_{t}+\Delta^{2} u-\Delta\left(u^{3}-u\right)=0$.

(B) Degenerate-mobility Cahn-Hilliard systems. The order parameter is once again $u=$ $\left(u^{1}, \ldots, u^{m}\right) \in \mathbb{R}^{m}$, but the interpretation is different: now $u^{i}$ represents the mass fraction of the $i$-th component in an $m$-component mixture. Therefore it must satisfy the pointwise constraint

$$
u^{i} \geqslant 0 \quad \text { for each } i, \quad \text { and } \quad \sum_{i=1}^{m} u^{i}=1 .
$$

The evolution preserves this constraint and decreases the free energy

$$
\int \frac{1}{2}|\nabla u|^{2}+\Psi(u) .
$$

At temperature $\theta$ the bulk term has the form

$$
\Psi(u)=\theta \sum_{i=1}^{m} u^{i} \ln u^{i}+u \cdot A u
$$

with $A=e \otimes e-I$, where $e=(1, \ldots, 1)$ and $I$ is the $m \times m$ identity. We shall focus on the deepquench limit, when $\theta=0$; then the preferred values of $u$-the system's phases—are the vertices of the constraint simplex 22]. The PDE has the form

$$
u_{t}^{i}=-\nabla \cdot J^{i}, \quad J^{i}=-\sum_{j=1}^{m} B_{i j}(u) \nabla w^{j},
$$

where the chemical potential $w^{i}$ is the first variation of the energy,

$$
w^{i}=\nabla_{u^{i}} \Psi(u)-\Delta u^{i},
$$

and the mobility $B_{i j}(u)$ is given by

$$
B_{i j}(u)=u^{i}\left(\delta_{i j}-u^{j}\right) .
$$

The special form of the mobility $B_{i j}$ assures that $\sum_{i} J^{i}=0$, and also that $u_{t}^{i}=0$ whenever $u^{i}=0$. Thus the evolution preserves the constraint (2). When $m=2$ this model reduces to the scalar degenerate-mobility Cahn-Hilliard equation: indeed, in that case $c=u^{1}-u^{2}$ satisfies $c_{t}=$ $\nabla \cdot\left(1-c^{2}\right) \nabla(-2 c-\Delta c)$.

The third model we study — from the epitaxial growth literature—is described near the end of this introduction.

For simplicity, we have presented in (B) only the simplest version of degenerate-mobility CahnHilliard dynamics. More general versions and references to the relevant physics literature can be found in [13]. In (A), by contrast, we made the opposite choice-presenting a very general PDE to which our method applies. The equations modeling spinodal decomposition are somewhat more restricted, since they come from free energies like (3) in the shallow-quench limit $(\theta$ just below the critical value $2 / m$ ). 
These PDE's have solutions. For the constant-mobility case, see e.g. [14] for a concise derivation of (11) and a global existence theorem. For the degenerate-mobility case, [13] proves the global existence of suitably-defined weak solutions for $\theta>0$, and remarks that the deep-quench limit $\theta \rightarrow 0$ can be treated as in the scalar case [12]. There is also some literature on the numerical approximation of these systems: see [2] and [3]. The PDE theory is far from complete; for example, the weak solutions constructed in [13] are not known to be unique. Fortunately, our method does not require smoothness or uniqueness; in particular it applies to the weak solutions constructed in [13, 14].

How should we expect solutions to behave as $t \rightarrow \infty$ ? The situation is analogous to the binary case, discussed at length in [22], so we shall be relatively brief. If we assume the solution coarsens, i.e.

$$
\frac{\text { interface width }}{\text { domain size }} \rightarrow 0
$$

then late-stage coarsening involves, more or less, a sharp-interface limit. The sharp-interface limit of (1) is a multicomponent version of Mullins-Sekerka dynamics [7]. The sharp-interface limit of (4) is a multicomponent version of motion by surface diffusion [17]. Both these results were demonstrated using asymptotic expansions; the paper [17] also includes local-in-time existence for the limiting geometric motion in 2D.

Each sharp-interface limit has a natural scale-invariance. Indeed, Mullins-Sekerka dynamics is invariant upon scaling space by $\lambda$ and time by $\lambda^{3}$; motion by surface diffusion is invariant upon scaling space by $\lambda$ and time by $\lambda^{4}$. If, as many people believe, coarsening is in some sense statistically self-similar, then scale-invariance determines the coarsening rate. We therefore expect the typical domain size to grow like $t^{1 / 3}$ for Mullins-Sekerka and $t^{1 / 4}$ for surface diffusion. And we expect the same for (1) and (4), since they can be viewed as diffuse-interface versions of the multicomponent Mullins-Sekerka and surface diffusion evolution laws.

For binary systems, modeled by scalar Cahn-Hilliard equations, the scaling and apparent selfsimilarity of coarsening has been studied in considerable depth: see e.g. [29] and the references given there. For multicomponent systems the literature on late-stage coarsening is however relatively sparse. One recent theme is the simulation of segregation dynamics for $q$-state Potts models with conserved kinetics: see [20, 28, 34] for the ternary case and [8] for larger $q$. The existence of a direct relation between Potts models and the continuous multicomponent PDE (1) is still open; but it is widely believed that the continuum limit of a Potts model should resemble a Cahn-Hilliard system [35]. (In the binary case, where the Potts model reduces to an Ising model, such a result is true: see e.g. [18].) The behavior of the Potts model is consistent with the conjectured coarsening rate for 11): the characteristic length scale is observed in the simulations to grow like $t^{1 / 3}$ for large $t$, until finite-size effects become important.

The main goal of the present paper is a rigorous bound on the coarsening rate. We shall prove, roughly speaking, that for (1) the typical length scale grows no faster than $t^{1 / 3}$, and for $(4)$ it grows no faster than $t^{1 / 4}$. Our analysis is the multicomponent extension of the argument in [22].

To define the "typical length scale" we need a scheme for averaging. Therefore it is convenient to solve the PDE's with periodic boundary conditions. The period cell $Q \subset \mathbb{R}^{n}$ is arbitrary, but we are mainly interested in the behavior when $Q$ is large; therefore it is important that all estimates be independent of the size of $Q$. We shall write $f_{Q} f$ for the average of a $Q$-periodic function $f$.

In studying phase separation, our interest is in solutions of (1) or (4) that represent mixtures of the pure phases. For such a solution, $u(x, t)$ should be near one of the zeros of the bulk term, except in some transition layers whose volume fraction is relatively small. Since the dynamics preserves 
$f_{Q} u$, the volume fractions of the phases are set by the initial data, and $f_{Q} u$ should lie in the convex hull of the pure phases.

The essential idea of [22] is to identify two distinct measures of the local length scale. The first is $1 / E$ where $E$ is the energy per unit volume. The second — call it $L$-is defined by a suitable negative norm of the order parameter. The main steps of the argument involve

(i) a "dissipation relation" linking $\dot{E}$ to $\dot{L}$;

(ii) an "isoperimetric inequality" constraining $E$ and $L$ pointwise; and

(iii) an ODE lemma, showing that (i) and (ii) imply a bound on the time-averaged coarsening rate.

Concerning (i): our notation (here and throughout the paper) is $\dot{f}=\partial f / \partial t$; the dissipation relation for 11 says $\dot{L}^{2} \leqslant-\dot{E}$. Concerning (ii): we shall prove that $E L \geqslant C$ for some constant $C$, provided $E$ is sufficiently small. We call this an isoperimetric inequality because the average energy $E$ resembles the perimeter of the interfaces. Concerning (iii): a special case says that if $E(t)$ and $L(t)$ are functions satisfying (a) $E L \gtrsim 1$ and (b) $\dot{L}^{2} \lesssim-\dot{E}$, then we have the following time-averaged lower bound on $E$ :

$$
f_{0}^{T} E^{2} \mathrm{~d} t \gtrsim f_{0}^{T}\left(t^{-1 / 3}\right)^{2} \mathrm{~d} t \quad \text { for } T \gg L_{0}^{3} \gg 1 \gg E_{0}
$$

Here $L_{0}=L(0), E_{0}=E(0)$, and the symbols $\gtrsim, \gg$ are to be interpreted as follows: $x \gtrsim y$ means $x \geqslant C y$ for some constant $C ; x \gg y$ means $x / y$ is sufficiently large. Thus (6) says there exists a constant $C>0$ (possibly very large), such that

$$
\frac{1}{T} \int_{0}^{T} E^{2} \mathrm{~d} t \geqslant \frac{1}{C} T^{-2 / 3}
$$

when $T \geqslant C L_{0}^{3}$ and $E_{0} \leqslant 1 / C$.

Our argument uses the same main steps. To capture the key issues, we briefly sketch it for the constant mobility case (1). The multicomponent versions of $E$ and $L$ are defined for any $Q$-periodic function $u=\left(u^{1}, \ldots, u^{m}\right)$ on $\mathbb{R}^{n}$ by

$$
E=f_{Q} \frac{1}{2}|\nabla u|^{2}+\Phi(u), \quad L=f_{Q}\left|\nabla^{-1} u\right|=\left(\sum_{i}\left(f_{Q}\left|\nabla^{-1} u^{i}\right|\right)^{2}\right)^{1 / 2}
$$

where

$$
f_{Q}\left|\nabla^{-1} u^{i}\right|=\sup _{g}\left\{f_{Q}\left(u^{i}-\bar{u}^{i}\right) g: g \text { periodic with sup }|\nabla g| \leqslant 1\right\}
$$

with $\bar{u}^{i}=f_{Q} u^{i}$.

With these choices of $E$ and $L$, the dissipation relation is relatively easy: the argument in [22] extends straightforwardly to the multicomponent setting. The isoperimetric inequality is however more subtle. Its statement is the same as in the scalar case, but the proof is significantly different. Our argument combines some elements from [22] and others from [1, 16, 33]. Once the dissipation relation and isoperimetric inequality are in hand no further work is required—our time-averaged bound on $E$ follows using the ODE lemma of [22].

Our method uses relatively little information from the phase-separation PDE's (1) and (4) basically just their dissipative, mass-conserving character. To emphasize this-and to give an 
additional application of the method-we also discuss the coarsening of a different class of fourthorder PDE's, proposed in the epitaxial growth literature as a model for the growth of some materials:

(C) An epitaxial growth model with anisotropy and slope selection. Here the unknown is a scalarvalued function $u$, representing the height of a growing film (more precisely: the difference between surface height and its mean). The PDE is

$$
u_{t}+\Delta^{2} u-\nabla \cdot\left(\nabla_{p} \Phi(\nabla u)\right)=0,
$$

which decreases the energy

$$
E=f_{Q} \frac{1}{2}|\nabla \nabla u|^{2}+\Phi(\nabla u)
$$

while conserving the mean $f u$. Our methods are applicable provided the bulk term $\Phi(\nabla u)$ "prefers" finitely many slopes $\nabla u \in\left\{\alpha_{1}, \ldots, \alpha_{k}\right\}$, i.e. provided $\Phi(\alpha)$ vanishes at these slopes and is positive otherwise. In applying the method, it is natural to assume that $u$ has mean value 0 , and to take $L$ as the standard deviation of the surface height

$$
L=\left(f_{Q} u^{2}\right)^{1 / 2} .
$$

Epitaxial growth models of this type are discussed for example in [24, 26, 32]. Space is twodimensional, and the preferred slopes $\alpha_{1}, \ldots, \alpha_{k}$ are usually assumed to be the vertices of a regular $k$-sided polygon in the plane. Heuristic arguments suggest a coarsening rate of $t^{1 / 3}$, at least when $k$ is large enough. Numerical simulations bear this out for $k=6$, but reveal much slower coarseningmore like $t^{1 / 4}$-when $k=4$. We shall show that the system can coarsen no faster than $t^{1 / 3}$. This is consistent with the behavior observed numerically, though it does not explain why $k=4$ is different. Our argument makes no use of symmetry — we assume nothing about the preferred slopes—and it works in any spatial dimension.

The isotropic analogue of 99 , associated with $\Phi(\nabla u)=\left(|\nabla u|^{2}-1\right)^{2}$, has also been considered as a model of epitaxial growth. It, too, coarsens no faster than $t^{1 / 3}$, as we show in [23]. But the proof is quite different from the one we give for 9 . . Briefly: the isoperimetric inequality is easier when there are finitely many preferred slopes, because each slope can be viewed as a distinct "phase." Unlike the present paper, the method of [23] is limited to space dimension two.

The paper is organized as follows: Section 2 addresses the constant mobility Cahn-Hilliard system (1); Section 3 addresses the epitaxial growth model 99; and Section 4 addresses the degenerate mobility Cahn-Hilliard system (4). Finally, in Section 5 we discuss some open problems.

\section{The constant mobility Cahn-Hilliard system}

This section addresses coarsening for the constant mobility Cahn-Hilliard system (1). Our structural hypotheses on the energy density $\Phi(u)$ are as follows:

A1. $\Phi \geqslant 0$, and $\Phi=0$ only at finitely many points $\left\{\alpha_{1}, \ldots, \alpha_{k}\right\} \subset \mathbb{R}^{m}$, with $k \geqslant 2$. Also, $\Phi$ is bounded away from zero outside a small neighborhood of each $\alpha_{i}$.

A2. There exist $a, \delta>0$ such that

$$
\frac{1}{a}\left|u-\alpha_{i}\right|^{2} \leqslant \Phi(u) \leqslant a\left|u-\alpha_{i}\right|^{2} \quad \text { when }\left|u-\alpha_{i}\right|<\delta .
$$


A3. There exist $b, R>0$ such that

$$
\Phi(u) \geqslant b|u|^{2} \quad \text { when }|u|>R .
$$

For example, $\Phi(u)$ could be $\min \left\{\left|u-\alpha_{1}\right|^{2}, \ldots,\left|u-\alpha_{k}\right|^{2}\right\}$.

Choosing $E$ and $L$ as in (7), we shall prove the following:

(i) Dissipation relation: $\dot{L}^{2} \leqslant-\dot{E}$.

(ii) Isoperimetric inequality: $E L \gtrsim 1$ when $E \ll 1$.

The constants implicit in (ii) depend on $\Phi$ through our structural hypotheses A1-A3. They are independent of $u$, provided that its mean value $\bar{u}$ stays bounded away from $\left\{\alpha_{1}, \ldots, \alpha_{k}\right\}$. This condition is natural: elementary examples show that the isoperimetric relation cannot be uniform in the limit as the mixture approaches a pure phase.

As explained in the introduction, (i) and (ii) lead immediately to the following time-averaged upper bound on the coarsening rate, using the framework of [22].

THEOREm 1 Suppose $0 \leqslant \theta \leqslant 1$ and $r<3$ satisfy $\theta r>0$ and $(1-\theta) r<2$. Fix $\rho>0$, and consider only initial data such that the mean $\bar{u}=f u$ satisfies $\left|\bar{u}-\alpha_{i}\right| \geqslant \rho$ for all $i$. Then there is a constant $C$ with the following property: for solutions of (1) on any period cell $Q \subset \mathbb{R}^{n}$, the energy per unit volume $E(t)$ and physical length scale $L(t)$ satisfy

$$
f_{0}^{T} E^{\theta r} L^{-(1-\theta) r} \mathrm{~d} t \geqslant \frac{1}{C} f_{0}^{T}\left(t^{-1 / 3}\right)^{r} \mathrm{~d} t \quad \text { for } T>C L_{0}^{3} \text {, provided } E_{0}<1 / C .
$$

Here $L_{0}=L(0)$ and $E_{0}=E(0)$. Moreover, the dependence of $C$ on $\Phi$ comes only from the constant in the isoperimetric inequality (ii).

Proof. Lemma 3 of [22] shows that [10] follows by an ODE argument once we know the two relations (i) and (ii).

The rest of this section is devoted to proving (i) and (ii).

The dissipation relation. In terms of the energy

$$
E(u)=f_{Q} \frac{1}{2}|\nabla u|^{2}+\Phi(u),
$$

one can write the system (1) in the form

$$
u_{t}^{i}+\nabla \cdot J^{i}=0, \quad J^{i}=-\nabla \frac{\delta E}{\delta u^{i}}, \quad i=1, \ldots, m .
$$

Thus

$$
-\dot{E}=-f_{Q} \sum_{i} \frac{\delta E}{\delta u^{i}} \frac{\partial u^{i}}{\partial t}=f_{Q} \sum_{i}\left|J^{i}\right|^{2}
$$

Recall from (7), (8) that

$$
L=\left(\sum_{i}\left(L_{i}\right)^{2}\right)^{1 / 2} \quad \text { with } \quad L_{i}=f_{Q}\left|\nabla^{-1} u^{i}\right| .
$$


Therefore $\dot{L}=L^{-1} \cdot \sum_{i} L_{i} \cdot \dot{L}_{i}$, and

$$
|\dot{L}| \leqslant L^{-1} L\left(\sum_{i}\left(\dot{L}_{i}\right)^{2}\right)^{1 / 2}=\left(\sum_{i}\left(\dot{L}_{i}\right)^{2}\right)^{1 / 2} .
$$

On the other hand, repeating the proof of Lemma 2 in [22], one obtains

$$
\left|\dot{L}_{i}\right| \leqslant f_{Q}\left|J^{i}\right| .
$$

The estimates (12) and (13) give

$$
\begin{aligned}
|\dot{L}| & \leqslant\left(\sum_{i}\left(\dot{L}_{i}\right)^{2}\right)^{1 / 2} \leqslant\left(\sum_{i}\left(f_{Q}\left|J^{i}\right|\right)^{2}\right)^{1 / 2} \\
& \leqslant\left(f_{Q} \sum_{i}\left|J^{i}\right|^{2}\right)^{1 / 2}=(-\dot{E})^{1 / 2},
\end{aligned}
$$

which is the desired relation.

The isoperimetric inequality. We must prove

$$
E L \gtrsim 1 \quad \text { when } E \ll 1
$$

with $E, L$ given by (7). This is asserted for any $Q$-periodic function $u$ whose mean satisfies $\left|\bar{u}-\alpha_{i}\right| \geqslant \rho$ for all $i$. The constants implicit in (15) depend on $\Phi$ and $\rho$ but not on the period cell $Q$. Notice that the condition $E \ll 1$ forces $\bar{u}$ to be in the convex hull of $\left\{\alpha_{1}, \ldots, \alpha_{k}\right\}$, or very close to its boundary.

The proof of (15) is essentially an interpolation argument. To simplify the notation we assume $\bar{u}=0$. (This represents no loss of generality, since we can always replace $u$ by $u-c$ and $\Phi(u)$ by $\Phi(u+c)$ with $c=\bar{u}$.) Our strategy is the following: we will construct a function $\phi: \mathbb{R}^{m} \rightarrow \mathbb{R}$ and a vector $\xi \in \mathbb{R}^{m}$ such that

B1. $|\phi(u)-\langle\xi, u\rangle| \lesssim \Phi^{1 / 2}(u)$,

B2. $|\nabla(\phi(u))| \lesssim \frac{1}{2}|\nabla u|^{2}+\Phi(u)$ a.e. in $Q$,

B3. when $E \ll 1, f_{Q}|\langle\xi, u\rangle| \gtrsim 1$.

Given such $\phi$ and $\xi$, the proof of the isoperimetric inequality is easy. Indeed, if $E \ll 1$, then B1 and B3 give

$$
f_{Q}|\phi(u)| \geqslant f_{Q}|\langle\xi, u\rangle|-C E^{1 / 2} \gtrsim 1
$$

Let $[\phi(u)]_{\varepsilon}$ denote the convolution of $\phi(u)$ with the kernel

$$
\frac{1}{\varepsilon^{n}} \eta\left(\frac{\dot{\varepsilon}}{\varepsilon}\right)
$$

where $\eta \geqslant 0$ is radially symmetric and supported in the unit ball with $\int_{\mathbb{R}^{n}} \eta=1$. We clearly have

$$
f_{Q}|\phi(u)| \leqslant f_{Q}\left|\phi(u)-[\phi(u)]_{\varepsilon}\right|+f_{Q}\left|[\phi(u)]_{\varepsilon}\right| .
$$


The first term is controlled by $\varepsilon E$, since

$$
\begin{aligned}
f_{Q}\left|\phi(u)-[\phi(u)]_{\varepsilon}\right| & \leqslant \sup _{|h| \leqslant \varepsilon} f_{Q}|\phi(u)(x)-\phi(u)(x+h)| \leqslant \varepsilon f_{Q}|\nabla \phi(u)| \\
& \lesssim \varepsilon f_{Q} \frac{1}{2}|\nabla u|^{2}+\Phi(u)=\varepsilon E
\end{aligned}
$$

using B2. The other term in (17) is controlled by $L / \varepsilon$; to see this, note first that

$$
\begin{aligned}
f_{Q}\left|[\phi(u)]_{\varepsilon}\right| & \lesssim f_{Q}\left|\left\langle\xi, u_{\varepsilon}\right\rangle\right|+f_{Q}\left|\left[\Phi^{1 / 2}(u)\right]_{\varepsilon}\right| \\
& \lesssim f_{Q}\left|u_{\varepsilon}\right|+E^{1 / 2}
\end{aligned}
$$

using B1. Since the convolution operator is symmetric in the $L^{2}$ norm and

$$
\sup \left|\nabla g_{\varepsilon}\right| \lesssim \frac{1}{\varepsilon} \sup |g| \quad \text { for any function } g
$$

a duality argument gives

$$
f_{Q}\left|u_{\varepsilon}\right| \lesssim \frac{1}{\varepsilon} f_{Q}\left|\nabla^{-1} u\right|=\frac{1}{\varepsilon} L
$$

using the convention $\bar{u}=0$. When $E \ll 1$, 16, 19 imply

$$
1 \lesssim \varepsilon E+\varepsilon^{-1} L
$$

The isoperimetric inequality follows by optimization over $\varepsilon$.

It remains to prove the existence of $\xi$ and $\phi$ satisfying B1-B3. The choice of $\xi$ is easy: it can be any vector such that $\left\langle\xi, \alpha_{j}\right\rangle \neq 0$ for each $j$. This assures that B3 is satisfied, since $E \ll 1$ implies that $u$ is close to $\left\{\alpha_{1}, \ldots, \alpha_{k}\right\}$ except on a set of small volume fraction. (We use here the hypothesis that $\bar{u}$ stays bounded away from the pure phases; after translating in $u$-space so that $\bar{u}=0$ this becomes the condition that $\left|\alpha_{i}\right| \geqslant \rho$ for each $i$.)

To prepare for the definition of $\phi$, let $d_{i}(u)$ be the geodesic distance from $\alpha_{i}$ to $u$ in the metric weighted by $\Phi^{1 / 2}$ :

$$
d_{i}(u)=\min \left\{\int_{0}^{1} \Phi^{1 / 2}(\gamma(t))\left|\gamma^{\prime}(t)\right| \mathrm{d} t: \gamma(0)=\alpha_{i}, \gamma(1)=u\right\}
$$

(see e.g. [1, 16, 33]). It is clearly Lipschitz continuous, with $\left|\nabla_{u} d_{i}\right| \leqslant \Phi^{1 / 2}$; therefore for any function $u(x)$ we have

$$
\left|\nabla_{x} d_{i}(u)\right| \leqslant \Phi^{1 / 2}(u)|\nabla u|
$$

Now let

$$
d_{\min }=\min _{i \neq j} d_{i}\left(\alpha_{j}\right), \quad \phi_{i}(u)= \begin{cases}d_{i}(u) & \text { if } d_{i}(u)<d_{\min } \\ d_{\min } & \text { otherwise }\end{cases}
$$


We claim that the function

$$
\phi(u)=\sum_{i=1}^{k} a_{i} \phi_{i}(u)
$$

satisfies $\mathrm{B} 1$ and $\mathrm{B} 2$ if the constants $a_{1}, \ldots, a_{k}$ are chosen appropriately. In fact, $\mathrm{B} 2$ holds for any choice of the $a_{i}$ 's, as an immediate consequence of 20). To get B1, it suffices to have

$$
\phi\left(\alpha_{i}\right)=\left\langle\xi, \alpha_{i}\right\rangle, \quad i=1, \ldots, k .
$$

To see why, notice that the left hand side of B1 is Lipschitz continuous with linear growth at infinity, while the right hand side of B2 vanishes only at the $\alpha_{i}$ 's, growing linearly (like $\left|u-\alpha_{i}\right|$ ) nearby and at least linearly at infinity. Therefore if 21 is true, B1 follows immediately.

Now, 211 gives a linear system of equations for $a_{1}, \ldots, a_{k}$; to complete the proof we need only check that the system is invertible. It has the form

$$
d_{\min }\left[\begin{array}{ccccc}
0 & 1 & 1 & \ldots & 1 \\
1 & 0 & 1 & \ldots & 1 \\
\vdots & & \vdots & & \vdots \\
1 & 1 & 1 & \ldots & 0
\end{array}\right]\left[\begin{array}{c}
a_{1} \\
\vdots \\
a_{k}
\end{array}\right]=\left[\begin{array}{c}
\left\langle\xi, \alpha_{1}\right\rangle \\
\vdots \\
\left\langle\xi, \alpha_{k}\right\rangle
\end{array}\right] .
$$

The matrix on the left is $e \otimes e-I$ with $e=(1, \ldots, 1)$, which is clearly invertible since its eigenvalues are -1 with multiplicity $k-1$ and $k-1$ with multiplicity 1 . So 21) has a solution and the proof is complete.

\section{The epitaxial growth model}

This section addresses the coarsening of the epitaxial growth model 99 with periodic boundary conditions. It represents steepest descent for

$$
E=f_{Q}|\nabla \nabla u|^{2}+\Phi(\nabla u) .
$$

Solutions are known to exist, globally in time (see e.g. [24]). As explained in the introduction, to apply our method it is natural to take

$$
L=\left(f_{Q} u^{2}\right)^{1 / 2} .
$$

We need not assume that $\bar{u}=0$, but this is the case of primary interest, since if $u$ solves the PDE so does $u-c$ for any constant $c$, and the $L^{2}$ norm is minimized when $c=\bar{u}$. Notice that when $\bar{u}=0$, $L$ is the standard deviation of $u$.

The dissipation relation is familiar: using the steepest-descent character of the PDE we get

$$
\dot{E}=-f_{Q} u_{t}^{2}, \quad L \cdot \dot{L}=f_{Q} u u_{t},
$$

whence

$$
\dot{L}^{2} \leqslant-\dot{E} .
$$


The isoperimetric inequality is also familiar, provided the preferred slopes $\alpha_{i}$ are all nonzero. Indeed, if $u$ is periodic then $v=\nabla u$ has mean value 0 , and

$$
f_{Q}\left|\nabla^{-1} v\right| \lesssim\left(f_{Q} u^{2}\right)^{1 / 2}
$$

Therefore if the $\alpha_{i}$ 's are nonzero, the isoperimetric inequality (15) gives

$$
\left(f_{Q} \frac{1}{2}|\nabla \nabla u|^{2}+\Phi(\nabla u)\right)\left(f_{Q} u^{2}\right)^{1 / 2} \gtrsim 1 \text { when } f_{Q} \frac{1}{2}|\nabla \nabla u|^{2}+\Phi(\nabla u) \ll 1 .
$$

As before, (23) and 24] imply a time-averaged lower bound on $E$ :

THEOREM 2 Consider periodic solutions of the epitaxial growth model (99), where the bulk energy $\Phi$ satisfies A1-A3 and the preferred slopes $\alpha_{i}$ are nonzero. Then for any $0 \leqslant \theta \leqslant 1$ and $r<3$ satisfying $\theta r>1$ and $(1-\theta) r<2$, there is a constant $C$ with the following property: for any period cell $Q \subset \mathbb{R}^{n}$, the energy per unit volume $E(t)$ and the standard deviation $L(t)$ satisfy

$$
f_{0}^{T} E^{\theta r} L^{-(1-\theta) r} \mathrm{~d} t \geqslant \frac{1}{C} f_{0}^{T}\left(t^{-1 / 3}\right)^{r} \mathrm{~d} t \quad \text { provided } \quad T \geqslant C L^{3}(0) \text { and } E(0) \leqslant \frac{1}{C} .
$$

\section{The degenerate mobility Cahn-Hilliard system}

This section addresses the degenerate mobility Cahn-Hilliard system (4) in the deep quench limit. In this case, the homogeneous part $\Psi$ of the free energy $E$ has the form

$$
\Psi(u)=u \cdot A u
$$

with $A=e \otimes e-I$, where $e=(1, \ldots, 1)$ and $I$ is the $m \times m$ identity. As explained in the introduction, the initial data should satisfy $0 \leqslant u^{i} \leqslant 1$ and $\sum_{i} u^{i}=1$ pointwise, and the equation preserves this constraint. We are interested in mixtures, so the mean of the initial data must have $\bar{u}^{i}$ bounded away from 1 for each $i$ (we permit $\bar{u}^{i}=0$ for some $i$-a mixture can use just some of the phases). Note that the mean $\bar{u}^{i}$ is preserved by the evolution (4).

As in the constant mobility case, we choose $E$ and $L$ by (7). We must prove a dissipation relation and an isoperimetric inequality. Focusing first on the latter, we assert that

$$
E L \gtrsim 1 \quad \text { when } E \ll 1 .
$$

This is not a trivial consequence of $(15)$ because the bulk energy $\Psi(u)$ does not satisfy A1-A3. A proof can be given by following the strategy we used for 15 . For variety, however, let us proceed differently, using the specific form of $\Psi$. Let $c^{i}=2 u^{i}-1$, so that $-1 \leqslant c^{i} \leqslant 1$, and $c^{i}$ is bounded away from both 1 and -1 for some $i$. Since $\sum_{i} u^{i}=1$, the energy can be expressed as

$$
E=f_{Q} \frac{1}{8}\left|\nabla c^{i}\right|^{2}+\frac{1}{4} \sum_{i=1}^{m}\left(1-\left(c^{i}\right)^{2}\right) .
$$

We shall use the scalar analogue of 15$)$ with $\Phi(c)=\left(c^{2}-1\right)^{2}$ : it asserts that for periodic functions $c^{i}(x)$ with $\bar{c}^{i}$ bounded away from \pm 1 ,

$$
\left(f_{Q} \frac{1}{2}\left|\nabla c^{i}\right|^{2}+\left(1-\left(c^{i}\right)^{2}\right)^{2}\right) \cdot f_{Q}\left|\nabla^{-1} c^{i}\right| \gtrsim 1 \text { when } f_{Q} \frac{1}{2}\left|\nabla c^{i}\right|^{2}+\left(1-\left(c^{i}\right)^{2}\right)^{2} \ll 1
$$


(This follows from (15); alternatively, it was proved in [22] for functions with mean value 0 , and the argument given there also proves 25 .) Since $\left|c^{i}\right| \leqslant 1$, we have

$$
f_{Q} \frac{1}{2}\left|\nabla c^{i}\right|^{2}+\left(1-\left(c^{i}\right)^{2}\right)^{2} \leqslant f_{Q} \frac{1}{2}\left|\nabla c^{i}\right|^{2}+\left(1-\left(c^{i}\right)^{2}\right) \ll 1 \quad \text { when } E \ll 1 .
$$

Therefore when $E \ll 1,(25)$ and $(26)$ give

$$
\left(f_{Q} \frac{1}{2}\left|\nabla c^{i}\right|^{2}+\left(1-\left(c^{i}\right)^{2}\right)\right) \cdot f_{Q}\left|\nabla^{-1} c^{i}\right| \gtrsim 1
$$

for any $i$ such that $\bar{c}^{i}$ stays bounded away from \pm 1 . The desired isoperimetric inequality is an immediate consequence.

The dissipation relation requires a bit more work. It says

$$
\dot{L}^{2} \lesssim-E \dot{E} .
$$

We present the argument for smooth solutions; the modifications required for weak solutions are minor, and are explained in [22]. From the dissipative structure of the equation we have

$$
-\dot{E}=\sum_{i, j=1}^{m} f_{Q} B_{i j}(u) \nabla \frac{\delta E}{\delta u^{i}} \nabla \frac{\delta E}{\delta u^{j}} .
$$

On the other hand, since $u_{t}^{i}=-\nabla \cdot J^{i}$ one verifies as in the constant-mobility case that

$$
\left|\dot{L}_{i}\right| \leqslant f_{Q}\left|J^{i}\right|
$$

for each $i$, so

$$
|\dot{L}| \leqslant\left(\sum_{i=1}^{m}\left(\dot{L}_{i}\right)^{2}\right)^{1 / 2} \lesssim \sum_{i=1}^{m}\left|\dot{L}_{i}\right| \leqslant \sum_{i=1}^{m} f_{Q}\left|J^{i}\right| .
$$

Since $J^{i}=-\sum_{j=1}^{m} B_{i j}(u) \nabla \frac{\delta E}{\delta u^{j}}$, we have

$$
\sum_{i=1}^{m}\left|J^{i}\right|=\max \left\{\sum_{k, i, j} B_{i j}(u) \nabla_{k} \frac{\delta E}{\delta u^{j}} \sigma_{k i}: \sum_{k} \sigma_{k i}^{2} \leqslant 1 \text { for each } i\right\} .
$$

Since the matrix $B_{i j}(u)$ is symmetric and nonnegative, we can apply the inequality $\langle B \xi, \eta\rangle \leqslant$ $\langle B \xi, \xi\rangle^{1 / 2}\langle B \eta, \eta\rangle^{1 / 2}$ to get

$$
\sum_{i, j} B_{i j}(u) \nabla_{k} \frac{\delta E}{\delta u^{j}} \sigma_{k i} \leqslant\left(\sum_{i, j} B_{i j}(u) \nabla_{k} \frac{\delta E}{\delta u^{i}} \nabla_{k} \frac{\delta E}{\delta u^{j}}\right)^{1 / 2} \cdot\left(\sum_{i, j}\left|B_{i j}(u) \sigma_{k i} \sigma_{k j}\right|\right)^{1 / 2} .
$$

Since $\sum_{k} \sigma_{k i}^{2} \leqslant 1$, we have $\left|\sigma_{k i}\right| \leqslant 1$ for each $k, i$, so

$$
\sum_{i, j}\left|B_{i j}(u) \sigma_{k i} \sigma_{k j}\right| \leqslant \sum_{i, j}\left|B_{i j}(u)\right| .
$$


Therefore summing over $k$ in $(29)$ and maximizing over $\sigma$ we conclude that

$$
\sum_{i=1}^{m}\left|J^{i}\right| \lesssim\left(\sum_{i, j} B_{i j}(u) \nabla \frac{\delta E}{\delta u^{i}} \nabla \frac{\delta E}{\delta u^{j}}\right)^{1 / 2} \cdot\left(\sum_{i, j}\left|B_{i j}(u)\right|\right)^{1 / 2} .
$$

Integrating, and using Hölder's inequality, we conclude that

$$
f_{Q} \sum_{i=1}^{m}\left|J^{i}\right| \lesssim\left(f_{Q} \sum_{i, j} B_{i j}(u) \nabla \frac{\delta E}{\delta u^{i}} \nabla \frac{\delta E}{\delta u^{j}}\right)^{1 / 2} \cdot\left(f_{Q} \sum_{i, j}\left|B_{i j}(u)\right|\right)^{1 / 2} .
$$

Since $\sum_{i} u^{i}=1$ we also have

$$
f_{Q} \sum_{i, j}\left|B_{i j}(u)\right|=f_{Q} \sum_{i}\left(u^{i}\left(1-u^{i}\right)+\sum_{j \neq i} u^{i} u^{j}\right)=f_{Q} \sum_{j \neq i} 2 u^{i} u^{j} \leqslant E .
$$

The desired dissipation relation is an immediate consequence of (28), 30, and (31).

Given these ingredients, the general framework of Kohn and Otto [22] implies the following theorem.

Theorem 3 Suppose $0 \leqslant \theta \leqslant 1$ and $r<4$ satisfy $\theta r>2$ and $(1-\theta) r<2$. Fix $\rho>0$, and consider only initial data for 4 that satisfy $\bar{u}^{i} \leqslant 1-\rho$ (in addition to the standard pointwise constraints $0 \leqslant u^{i} \leqslant 1$ and $\sum_{i} u^{i}=1$ ). Then

$$
f_{0}^{T} E^{\theta r} L^{-(1-\theta) r} \mathrm{~d} t \gtrsim f_{0}^{T}\left(t^{-1 / 4}\right)^{r} \mathrm{~d} t \quad \text { for } T \gg L_{0}^{4} \gg 1 \gg E_{0} .
$$

\section{Discussion}

Our bounds are rather weak: they control only time-averaged value of $E$, and they do not control $L$. The situation is the same as in [22,23]: the method of Kohn and Otto seems unable to do better. It would be nice to prove pointwise bounds on $E$ and $L$, but this seems to require a different method.

The problems considered here involve finitely many "phases." The analogous problems with a continuum of preferred states are fundamentally different. Consider for example what becomes of our constant-mobility Cahn-Hilliard system $\sqrt{1}$ when the bulk term is $\Phi(u)=\left(1-|u|^{2}\right)^{2}$ with $u \in \mathbb{R}^{m}$. The energy per unit area is then

$$
E=f \frac{1}{2}|\nabla u|^{2}+\left(1-|u|^{2}\right)^{2}
$$

and the preferred values of $u$ form a continuum-namely the unit sphere. The evolution equation (1) becomes

$$
u_{t}-\nabla \cdot\left(\nabla \frac{\delta E}{\delta u}\right)=0
$$

which decreases the energy while conserving the mean of $u$. The associated coarsening has been considered in [5, 6, 31]. Heuristic arguments suggest that $L(t) \sim t^{1 / 4}$ for all spatial dimensions $n \geqslant 2$ and order-parameter dimensions $m \geqslant 2$; this has been confirmed numerically in certain 
cases [5, 20, 31]. We wonder whether the viewpoint of the present paper might be applicable to this problem.

For the energy (32), the case $m=n=2$ seems special. Indeed, when the order parameter $u$ and the spatial variable $x$ are both two-dimensional the coarsening process involves the interaction and cancellation of vortices. To explain why, suppose the unit cell $Q$ has side-length $1 / \varepsilon$, and let $\psi(x)=u(x / \varepsilon)$. Then the energy per unit area becomes

$$
E=\varepsilon \int_{Q_{1}} \frac{\varepsilon}{2}|\nabla \psi|^{2}+\varepsilon^{-1}\left(1-|\psi|^{2}\right)^{2} .
$$

The asymptotic behavior of this functional as $\varepsilon \rightarrow 0$ has been studied at length: see e.g. [4]. The asymptotics of the associated second-order, steepest-descent dynamics $u_{t}+\delta E / \delta u=0$ have also been studied: see e.g. [21, 25, 30]. But the analysis of coarsening is an entirely different type of question. Given an initial condition with many vortices (randomly placed, perhaps, with random degrees), the evolution makes the vortices interact and eventually annihilate one another. In discussing coarsening, we want to know the density of vortices that remain at time $t$. Can the method of this paper be used to bound the coarsening rate?

Another open problem was noted in Section 3. It concerns the epitaxial growth model in two space dimensions, when the preferred values of $\nabla u$ are the vertices of a square. The observed coarsening rate is slower than $t^{1 / 3}$ in this case [26]. The reason appears to be that the valleys and peaks in the graph of $u$ form a lattice with defects-and the true coarsening mechanism is the motion of these defects. We wonder whether this intuition can be turned into a rigorous proof that the coarsening rate is strictly slower then than $t^{1 / 3}$.

Acknowledgements. We thank Amy Novick-Cohen for comments on an earlier version of this paper. This research was partially supported by NSF grant DMS 0073047.

\section{REFERENCES}

1. BALdo, S. Minimal interface criterion for phase transitions in mixtures of Cahn-Hilliard fluids. Ann. Inst. H. Poincaré Anal. Non Linéaire 7 (1990), 67-90. Zbl 0702.49009 MR 91j:76005

2. Barrett, J. W. \& Blowey, J. F. An improved error bound for a finite element approximation of a model for phase separation of a multi-component alloy with a concentration dependent mobility matrix. Numer. Math. 88 (2001), 255-297. Zbl 0990.65105 MR 2002c:65162

3. Barrett, J. W., Blowey, J. F., \& Garcke, H. On fully practical finite element approximations of degenerate Cahn-Hilliard systems. M2AN Math. Model. Numer. Anal. 35 (2001), 713-748. Zbl 0987.35071 MR 2002k:65149

4. Bethuel, F., Brezis, H., \& HÉlein, F. Ginzburg-Landau Vortices. Birkhäuser, Boston (1994).

5. J. BRAY, A. Theory of phase-ordering kinetics. Adv. Phys. 43 (1993), 357-459. Zbl 0802.35142 MR 95c:58044

6. Bray, A. \& Rutenberg, A. Energy-scaling approach to phase ordering growth laws. Phys. Rev. E 51 (1995), 5499-5514.

7. Bronsard, L., Garcke, H., \& Stoth, B. A multi-phase Mullins-Sekerka system: Matched asymptotic expansions and an implicit time discretisation for the geometric evolution problem. Proc. Roy. Soc. Edinburgh Ser. A 128 (1998), 481-506. Zbl 0924.35199 MR 2000e:35227

8. DAS, S. K. \& PURI, S. Dynamics of phase separation in multicomponent mixtures. Phys. Rev. E 65 (2002), 026141-026149. 
9. DeFontaine, D. A computer simulation of the evolution of coherent composition variations in solid solutions. Ph.D. thesis, Northwestern Univ., Evansville, IL, 1967.

10. DeFontaine, D. An analysis of clustering and ordering in multicomponent solid solutions, I. Stability criteria. J. Phys. Chem. Solids 33 (1972), 297-310.

11. DeFontaine, D. An analysis of clustering and ordering in multicomponent solid solutions, II. Fluctuations and kinetics. J. Phys. Chem. Solids 34 (1973), 1285-1304.

12. Elliott, C. \& Garcke, H. On the Cahn-Hilliard Equation with degenerate mobility. SIAM J. Math. Anal. 27 (1996), 404-423. Zbl 0856.35071 MR 97c:35081

13. Elliott, C. \& Garcke, H. Diffusional phase transitions in multicomponent systems with a concentration dependent mobility matrix. Phys. D 109 (1997), 242-256. Zbl 0925.35087 MR 99b:35107

14. Elliott, C. \& Luckhaus, S. A generalized diffusion equations for phase separation of a multicomponent mixture with interfacial free energy. IMA Preprint 887 (1991).

15. Eyre, D. Systems of Cahn-Hilliard equations. SIAM J. Appl. Math. 53 (1993), 1686-1712. Zbl 0853.73060 MR 94j:73010

16. FONSECA, I. \& TARTAR, L. The gradient theory of phase transitions for systems with two potential wells. Proc. Roy. Soc. Edinburgh Ser. A 111 (1989), 89-102. Zbl 0676.49005 MR 90b:49021

17. Garcke, H. \& Novick-Cohen, A. A singular limit for a system of degenerate Cahn-Hilliard equations. Adv. Differential Equations 5 (2000), 401-434. Zbl 0988.35019 MR 2001e:35097

18. Giacomin, G., Lebowitz, J. L., \& Presutti, E. Deterministic and stochastic hydrodynamic equations arising from simple microscopic model systems. Stochastic Partial Differential Equation: Six Perspectives, R. A. Carmona and B. Rozovskii (eds.), Math. Surveys Monogr. 44, Amer. Math. Soc. (1997), 107-152. Zbl 0927.60060 MR 2000f:60151

19. Grant, C. Slow motion in one-dimensional Cahn-Morral system. SIAM J. Math. Anal. 26 (1995), 2134. Zbl 0813.35042 MR 95i:35247

20. Jeppesen, C. \& Mouritsen, O. G. Universality of ordering dynamics in conserved multicomponent systems. Phys. Rev. B 47 (1993), 14724-14733.

21. Jerrard, R. \& Soner, M. Dynamics of Ginzburg-Landau vortices. Arch. Rat. Mech. Anal. 142 (1998), 99-125. Zbl 0923.35167 MR 99m:58073

22. Kohn, R. V. \& Отто, F. Upper bounds on coarsening rates. Comm. Math. Phys. 229 (2002), 375-395. Zbl 1004.82011 MR 1924360

23. Kohn, R. V. \& YAN, X. Upper bounds on the coarsening rate for an epitaxial growth model. Comm. Pure Appl. Math. 56 (2003), 1549-1564. ZZbl pre01997827] MR 1995869

24. LI, B. \& LiU, J. Thin film epitaxy with or without slope selection. Euro. J. Appl. Math., to appear.

25. LIN, F. Some dynamical properties of Ginzburg-Landau vortices. Comm. Pure Appl. Math. 49 (1996), 323-359. Zbl 0853.35058 MR 97c:35189

26. Moldovan, D. \& Golubovic, L. Interfacial coarsening dynamics in epitaxial growth with slope selection. Phys. Rev. E 61 (2000), 6190-6214.

27. Morral, J. E. \& Cahn, J. W. Spinodal decomposition in ternary systems. Acta Metall. 19 (1971), $1037-1045$.

28. Okabe, Y. Application of the exchange Monte Carlo method to ordering dynamics. New J. Phys. 1 (1999), 10.1-10.7.

29. Puri, S., Bray, A. J., \& Lebowitz, J. L. Phase-separation kinetics in a model with order-parameterdependent mobility. Phys. Rev. E 56 (1997), 758-765.

30. Rubinstein, J. \& Sternberg, P. On the slow motion of vortices in the Ginzburg-Landau heat flow. SIAM J. Math. Anal. 26 (1995), 1452-1466. Zbl 0838.35102 MR 96j:35239

31. Siegert, M. Non-equilibrium ordering dynamics and pattern formation. Scale Invariance, Interfaces, and Non-equilibrium Dynamics, A. J. McKane et al. (eds.), NATO ASI Ser. B Phys. 344 (1995), 165-202. 
32. Siegert, M. Ordering dynamics of surfaces in molecular beam epitaxy. Phys. A 239 (1997), 420-427.

33. Sternberg, P. Vector-valued local minimizers of nonconvex variational problems. Rocky Mountain J. Math. 21 (1991), 799-807. Zbl 0737.49009 MR 92e:49016

34. Tafa, K., Puri, S., \& Kumar, D. Kinetics of phase separation in ternary mixtures. Phys. Rev. E 64 (2001), 056139-050148.

35. Wu, F. The Potts model. Rev. Mod. Phys. 54 (1982), 235-268. 